\title{
Objektverfolgung bei einer vollständigen Fahrzeugumfeldüberwachung mittels Radar
}

\author{
M. Schuster ${ }^{1,2}$, T. Pech ${ }^{2}$, J. Reuter ${ }^{1}$, and G. Wanielik ${ }^{2}$ \\ ${ }^{1}$ Institut für Systemdynamik, HTWG Konstanz, Deutschland \\ ${ }^{2}$ Professur für Nachrichtentechnik, TU Chemnitz, Deutschland \\ Correspondence to: M. Schuster (Michael.Schuster@htwg-konstanz.de)
}

Received: 26 January 2014 - Revised: 17 June 2014 - Accepted: 29 June 2014 - Published: 10 November 2014

\begin{abstract}
Kurzfassung. Für modernste Fahrerassistenzsysteme ist zunehmend eine vollständige Rundumüberwachung des flieBenden Verkehrs erforderlich. In diesem Artikel wird ein Ansatz zur Überwachung des gesamten umliegenden Verkehrs mittels Radarsystemen präsentiert. Hierfür wurde ein Versuchsfahrzeug mit insgesamt sechs Radarsensoren ausgerüstet, um eine möglichst hohe Umfeld-Überwachung zu ermöglichen. Für diese Konfiguration wird ein auf GPDA basierendes Trackingsystem vorgestellt. Es wird auf das Problem ausgedehnter Ziele eingegangen, welches auftritt wenn sich ein Fremdfahrzeug neben dem Versuchsfahrzeug befindet, da hier Reflektionen von verschiedenen Reflektionszentren des Ziels aufgefangen werden. Dieser Effekt wird ausgenutzt, um mittels einer Track-zu-Track Fusion eine Längenschätzung des vorbeifahrenden Fahrzeugs zu ermöglichen.
\end{abstract}

\section{Einführung}

Die Verwendung von Radarsystemen für Fahrerassistenzsysteme ist inzwischen zum Standard für viele Anwendungen geworden. So sind beispielsweise der Abstandsregeltempomat (Adaptive Cruise Control) mit einem im vorderen Stoßfänger integrierten Radar seit vielen Jahren in Verwendung. Auch sind in vielen Fahrzeugen inzwischen Kurzstreckenradare eingebaut, welche den Fahrer darüber informieren, ob sich im toten Winkel ein anderer Verkehrsteilnehmer befindet.

Diese Systeme arbeiten bisher unabhängig von einander. Für einen Spurwechselassistenten, welcher den optimalen Zeitpunkt für einen Spurwechsel prädizieren soll, sind jedoch Informationen über vorausfahrenden und rückwärtigen Verkehr als auch über sich auf angrenzenden Fahrstreifen befindliche Fahrzeuge erforderlich (Schubert et al., 2010). Für diese Anwendung oder auch für vollständig autonome Fahrzeuge werden in der Regel eine Vielzahl verschiedener Sensortypen eingesetzt (Darms et al., 2009). Da jedoch insbesondere Lasersysteme sehr teuer sind, wird in dieser Arbeit ein System zur Rundumüberwachung realisiert, welches primär mit Radarsensoren arbeitet.

$\mathrm{Zu}$ diesem Zweck wurde ein Versuchsfahrzeug mit insgesamt sechs Radarsensoren ausgestattet. Alle Sensoren übertragen ihre Detektionen an ein gemeinsames TrackingSystem, in dem die erhaltenen Messungen asynchron verarbeitet werden können.

In diesem Artikel werden zunächst die eingesetzten Sensoren und die Systemarchitektur kurz vorgestellt und auf das sich ergebende Problem der ausgedehnten Ziele eingegangen. Im dritten Kapitel wird das verwendete Trackingverfahren auf Basis des Generalized Probabilistic Data Association (GPDA) Filters erläutert, sowie einige Implementierungsdetails vorgestellt. Im vierten Kapitel werden praktische Ergebnisse gezeigt und ein Ansatz zur Schätzung der Größe eines Fahrzeugs auf Basis von Trackfusion gezeigt.

\section{Systemarchitektur und Problembeschreibung}

Das Versuchsfahrzeug wurde mit zwei verschiedenen Radarsystemen ausgestattet (Abb. 1). In der Front und im Heck des Fahrzeugs ist jeweils mittig ein $77 \mathrm{GHz}$-Fernbereichssensor mit einem maximalen Detektionswinkel von $\pm 28^{\circ}$ verbaut. An den Seiten des Fahrzeugs befinden sich je zwei $24 \mathrm{GHz}-$ Breitbandsysteme. Diese verfügen über eine hohe Entfernungsauflösung und einen Abdeckungsbereich von ca. $80^{\circ}$. Jedoch ist die Reichweite auf höchstens $10 \mathrm{~m}$ begrenzt.

Die Anordnung der Radarsensoren ermöglicht eine fast geschlossene $360^{\circ}$ Umfelderkennung für Fremdfahrzeuge, 


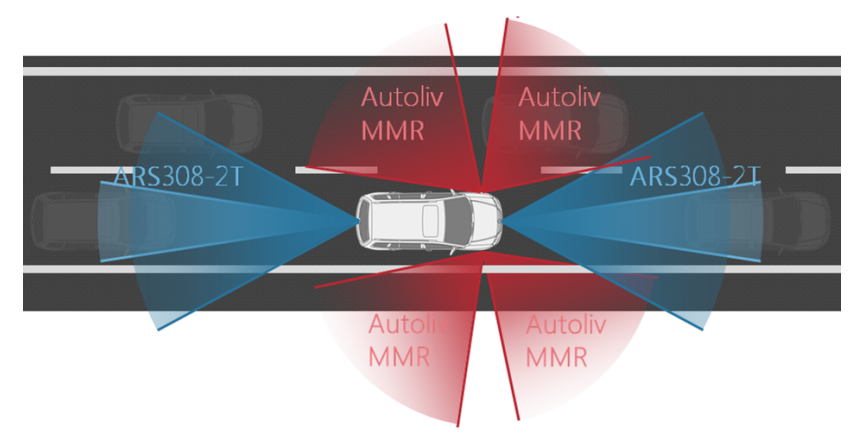

Abb. 1. Einbaupositionen und Sichtfelder der $77 \mathrm{GHz}$ Radare (blau) und der $24 \mathrm{GHz}$ Radare (rot)

welche sich vor, neben und hinter dem Eigenfahrzeug befinden. Jedoch gibt es hinter dem Heck einen Bereich von wenigen Metern, wo eine Detektion kleiner Fahrzeuge nicht möglich ist. Abbildung 2 zeigt die typische Lage von Messpunkten während eines Überholvorgangs durch ein Zielfahrzeug.

Die Lage der Detektionen auf dem Zielfahrzeug ist dabei stark abhängig vom Erfassungswinkel des jeweiligen Sensors. Zu Beginn des Manövers liegen die Detektionen vorwiegend auf der Front des Zielfahrzeugs. Im Verlauf des Manövers stammen diese dann von den Radläufen bzw. zum Ende des Manövers nur noch vom Heck des Überholenden. Dieser Übergang erfolgt dabei nicht kontinuierlich sondern relativ abrupt, sowohl beim Übergang von einem Sensorsichtbereich in den nächsten, als auch innerhalb eines Sensorbereiches. Dies liegt daran, dass ein Fahrzeug in der Regel aus einer Vielzahl von Radarreflektoren besteht. Aufgrund der begrenzten Sensorauflösungen werden in der Regel mehrere Reflektoren zu einem Reflektionscluster zusammengefasst und als eine Detektion gemeldet. Je nach Erfassungswinkel und Entfernung zum Ziel können auch mehrere Reflektionscluster erfasst werden. Dies ist insbesondere bei der Passage der Seitenradare der Fall. Ein Simulationsmodell zur Prädiktion dieser Detektionslisten wurde u.a. in Buhren and Yang (2006) vorgestellt.

\section{Tracking framework}

Hauptaufgabe der Multi-Objektverfolgung ist die Schätzung von Position und Bewegungsrichtung aller vorhandenen $\mathrm{Ob}-$ jekte sowie die Ermittlung der Wahrscheinlichkeit, dass überhaupt ein Objekt an dieser Stelle existiert. In diesem Abschnitt wird das Verfahren des Assoziationsalgorithmus erläutert und einige wichtige Details zur Implementierung aufgeführt.

Eines der Hauptprobleme der Multi-Objekt Verfolgung ist die Zuweisungsproblematik. In der Regel ist nicht bekannt, welche der empfangenen Detektionen von welchem der vorhandenen Objekte stammt. Insbesondere bei Radarsystemen

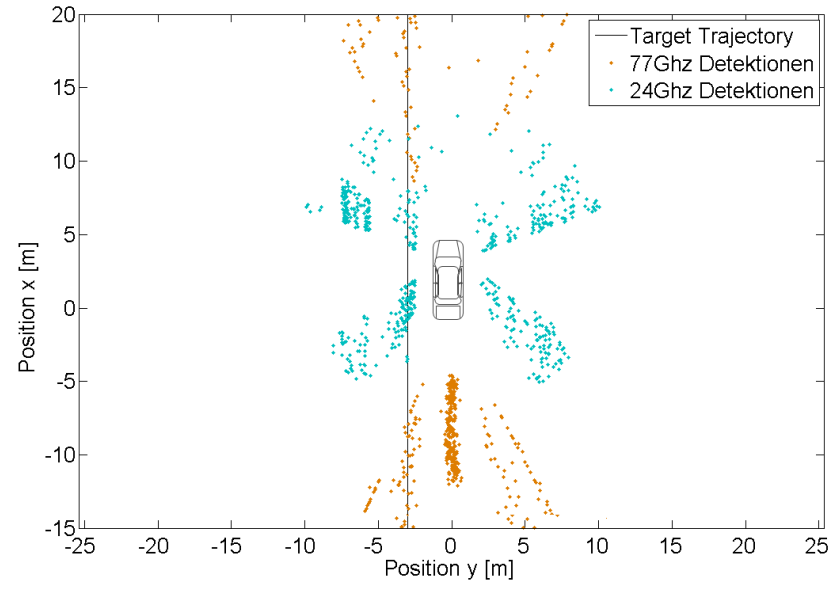

Abb. 2. Detektionen der $77 \mathrm{Ghz}$ Radare (orange) und der $24 \mathrm{GHz}$ Radare (blau) während eines Überholmanövers

wird dies noch durch gemeldete Detektionen, welche ohne ein real existierendes Objekt entstanden sind erschwert (Clutter). Auch ist es möglich, dass ein vorhandenes Objekt überhaupt nicht detektiert wird. In der beschriebenen Applikation ist dies insbesondere der Fall, wenn sich innerhalb des Detektionsbereichs eines Seitenradars nur Flächenreflektoren (bspw. Fahrzeugtüren) befinden, da diese nur unter einem rechten Winkel ein Echo an das Radar zurück senden.

Vom Prinzip her kann man die bekannten Assoziationsverfahren in zwei Gruppen einteilen. Die erste Gruppe verwendet für jedes Objekt einen eigenen Filter. Für jeden dieser Filter muss dann eine explizite Zuweisung von Messungen erfolgen. Bekannteste Vertreter dieser Gattung sind das Nearest Neighbour, Probabilistic Data Association und Multi-Hypothesen Verfahren (Blackman and Popoli, 1999). In den letzten Jahren hat noch eine zweite Gruppe eine verstärkte Aufmerksamkeit erfahren. Diese verwendet nur eine Filterinstanz für alle Objekte. In diesem Ansatz wird der Zustand aller Objekte durch eine gemeinsame multimodale Wahrscheinlichkeitsdichte beschrieben. Hierdurch wird es beispielsweise möglich auch Interaktionen zwischen Objekten zu modellieren. Jedoch kann auch der Rechenaufwand erheblich steigen, da häufig Sequential Monte Carlo-Methoden zum Einsatz kommen. Bekanntester Vertreter dieser Art von Filter ist das Probability Hypothesis Density Filter (Erdinc et al., 2005).

Für die in Kapitel zwei beschriebene Applikation können folgende Annahmen getroffen werden:

- Es können mehrere Detektionen von einem Fahrzeug empfangen werden.

- Einzelne Objekte kreuzen ihre Fahrspuren nur mit ausreichend räumlichen Abstand bezogen auf die Radarauflösung.

Auf Basis der getroffenen Annahmen wurde das von Schubert et al. (2012) vorgestellte Generalized Probabilistic Data 


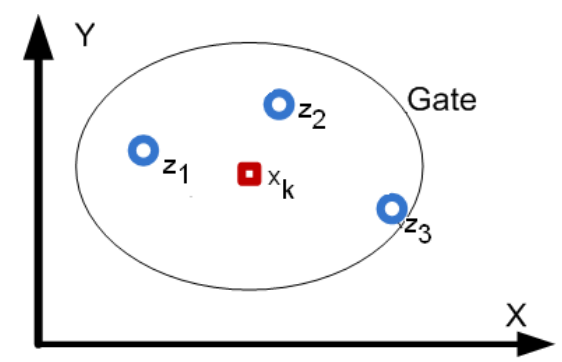

Abb. 3. Empfangene Messungen $\mathbf{z}_{k}$ (blau) innerhalb des Einzugsbereichs von Track $\boldsymbol{x}_{k}$ (rot)

Association (GPDA) Verfahren gewählt. Das GPDA stellt eine verallgemeinerte Form des seit vielen Jahren etablierten Integrated Probabilistic Data Association (IPDA) Verfahrens dar (Musicki et al., 1994). Dieses Verfahren ordnet alle Messungen die sich im Umkreis um ein Objekt befinden diesem zu (Abb. 3). Für jede Messung wird eine Assoziationswahrscheinlichkeit berechnet, unter der Annahme, dass genau eine Messung von diesem Objekt stammt. Dabei wird auch die Hypothese berücksichtigt, dass keine Messung von diesem Objekt stammt. Das IPDA berechnet zudem die Existenzwahrscheinlichkeit für dieses Objekt und berücksichtigt diese bei der Messdatenzuordnung.

\subsection{Generalized probabilistic data association}

Das GPDA erweitert das IPDA um die Annahme, dass bis zu $n$-Messungen von einem Objekt stammen können. Die posteriori-Wahrscheinlichkeitsdichte für einen Objektzustand $\boldsymbol{x}_{k}$ für einen Datensatz mit $\mathbf{Z}^{k}$ Messungen bis zum Zeitpunkt $k$, wird definiert mit

$p\left(\boldsymbol{x}_{k} \mid \mathbf{Z}^{k}\right)=\sum_{A_{i}^{m} \in A} p\left(\boldsymbol{x}_{k} \mid \mathbf{Z}_{k}, A_{i}^{m}\right) P\left(A_{i}^{m} \mid \mathbf{Z}_{k}\right)$.

Durch das Set $A$ werden alle möglichen Zuordnungshypothesen beschrieben. $A^{m}$ beschreibt die Untergruppe, dass Track $\boldsymbol{x}$ insgesamt $m$ von $n$ Messungen zugeordnet werden. Der Parameter $n$ kann ein vordefinierter Systemparameter sein. Für den Fall dass die Anzahl der verfügbaren Messungen $n_{z}$ zum Zeitpunkt $k$ kleiner als $n$ ist, wird $n=n_{z}$ gesetzt. Für den Regelfall $n_{z}>n$ ergeben sich folgende Assoziationshypothesen:

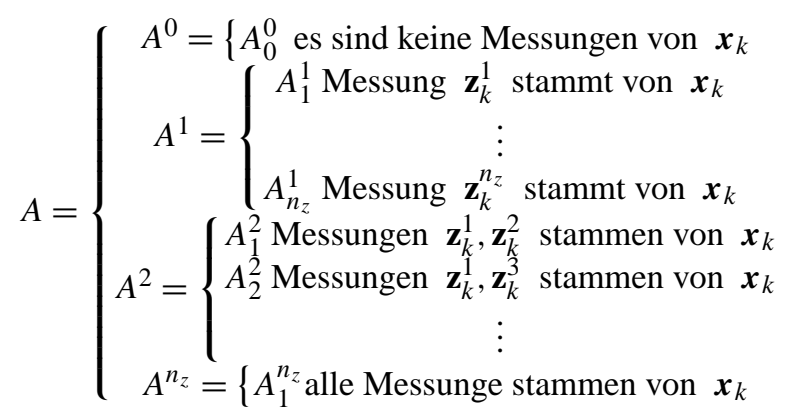

Für die sensorspezifische Zuordnung einer Messung zu einem vorhanden Objekt wird die Wahrscheinlichkeit $\Lambda_{k}^{i}=$ $p\left(\mathbf{z}_{k}^{i} \mid \mathbf{Z}_{k}\right)$ definiert. Da a-priori nichts über die Anzahl der Messungen bekannt ist, wird diese als gleich verteilt angenommen. Die Clutter-Dichte wird als Poissonverteilung mit Parameter $\lambda$ angenommen. Dieser beschreibt das Verhältnis von Anzahl der Falschmessungen zum überwachten Raum. Mit diesen Annahmen lassen sich die Assoziationswahrscheinlichkeiten $P\left(A_{i}^{m} \mid \mathbf{Z}_{k}\right)$ in Abhängigkeit der Detektionswahrscheinlichkeit $P_{\mathrm{D}}$ und der Existenzwahrscheinlichkeit $P_{\mathrm{E}}$ wie folgt definieren:

Der Fall, dass keine Messung von diesem Objekt stammt hat die Wahrscheinlichkeit

$P\left(A_{0}^{m} \mid \mathbf{Z}_{k}\right)=\frac{1-P_{\mathrm{D}} P_{\mathrm{E}}}{1-\delta P_{\mathrm{E}}}$

und für die $i$-te Messung gilt

$$
P\left(A_{i}^{m} \mid \mathbf{Z}_{k}\right)=\frac{P_{\mathrm{D}} P_{\mathrm{E}} n_{z}^{\frac{m}{z}} \lambda^{-m}}{n-\delta P_{\mathrm{E}}} \prod_{j=1}^{n_{z}}\left(\Lambda_{k}^{i}\right)^{C_{n_{z}}^{m}[i, j]} .
$$

Die Hilfsvariable $\delta$ is definiert als

$$
\delta=P_{\mathrm{D}}-\frac{P_{\mathrm{D}}}{n} \sum_{m=1}^{\min \left(n, n_{z}\right)} n_{z}^{\frac{m}{z}} \cdot \lambda^{-m} \sum_{i=0}^{\left(\begin{array}{c}
n_{z} \\
m
\end{array}\right)} \prod_{j=1}^{n_{z}}\left(\Lambda_{k}^{i}\right)^{C_{n_{z}}^{m}[i, j]} .
$$

mit

$n_{z}^{\frac{m}{z}}=\frac{n_{z} !}{\left(n_{z}-m !\right)}$.

Der Ausdruck $C_{n_{z}}^{m}[i, j] \in\{0,1\}$ beschreibt die Zuordnung der $j$-ten Messung aus der Kombination $i$ des Hypothesensatz $A^{m}$.

Die bedingte posteriori Wahrscheinlichkeitsdichte $p\left(\boldsymbol{x}_{k} \mid \mathbf{Z}_{k}, A_{i}^{m}\right)$ kann nun leicht durch bspw. Verwendung eines Kalman Filters ermittelt werden. Die Filterergebnisse werden gemäß der Assoziationswahrscheinlichkeiten gewichtet und aufsummiert.

Die Existenzwahrscheinlichkeit des Objekts wird durch

$P\left(\exists x_{k}=\exists \mid \mathbf{Z}_{k}\right)=\frac{(1-\delta) P_{\mathrm{E}}}{\left(1-\delta P_{\mathrm{E}}\right)}$

aktualisiert.

\subsection{Modellbildung}

Das Bewegungsmodell eines Objekts wird in der Form

$\boldsymbol{x}_{k+1}=f_{k}\left(\boldsymbol{x}_{k}\right)+w_{k}$

angesetzt. Für die Transitionsfunktion $f_{k}$ wird das Constant Turn Rate and Velocity (CTRV) Modell gewählt (Schubert et al., 2008). Der Zustandsvektor ist definiert durch $\boldsymbol{x}=$ $[x, y, \psi, r, V]$ mit $x, y$ als Position im körperfesten Koordinatensystem des Versuchsfahrzeugs. Weiter ist $\psi$ der Kurswinkel, $r$ ist die zugehörige Drehrate und $V$ beschreibt die 
horizontale Geschwindigkeit. Die Unsicherheit in der wahren Bewegung zwischen zwei Zeitschritten $w_{k}$ wird durch zwei normalverteilte Zufallszahlen mit Mittelwert 0 und Standardabweichungen $\sigma_{r}, \sigma_{V}$ berücksichtigt, welche kleine Änderungen in der Drehrate und der Geschwindigkeit repräsentieren.

Jede Detektion $z^{i}=[r, \phi, \dot{r}]$ eines Sensors besteht aus Entfernung zum Ziel $r$, dem Detektionswinkel $\phi$ und der Annäherungsgeschwindigkeit $\dot{r}$. Damit folgt für das Messmodel $z_{k}^{i}=h\left(\boldsymbol{x}_{k}\right)+v_{k}$ die Beziehung

$h\left(\boldsymbol{x}_{k}\right)=\left\{\begin{array}{c}\sqrt{x_{\mathrm{s}}^{2}+y_{\mathrm{s}}^{2}} \\ \operatorname{atan} \frac{y_{\mathrm{s}}}{x_{\mathrm{s}}} \\ \frac{x_{\mathrm{s}} \dot{x}_{\mathrm{s}}+y_{\mathrm{s}} y_{\mathrm{s}}}{\sqrt{x_{\mathrm{s}}^{2}+y_{\mathrm{s}}^{2}}}\end{array}\right.$.

Da sich die Sensoren an verschiedenen Punkten des Versuchsfahrzeugs mit verschiedenen Einbauwinkeln befinden, muss zur Vorhersage des Messdatums zunächst die Position und die Geschwindigkeit des Objekts in das Sensorkoordinatensystem transformiert werden:

$$
\begin{aligned}
& x_{\mathrm{s}}=\Delta x \cos \left(\theta_{i}\right)+\Delta y \sin \left(\theta_{i}\right) \\
& y_{\mathrm{s}}=-\Delta x \sin \left(\theta_{i}\right)+\Delta y \cos \left(\theta_{i}\right)
\end{aligned}
$$

Hierin ist $\theta_{i}$ der Einbauwinkel des $i$-ten Sensor und $\Delta x, \Delta y$ die Differenz zwischen Position des Objekts und der Einbauposition des jeweiligen Sensors. Ebenso muss zur Berechnung der Annäherungsgeschwindigkeit die horizontale Objektgeschwindigkeit um die Eigengeschwindigkeit $V_{\mathrm{E}}$ des Versuchsfahrzeugs kompensiert werden:

$$
\begin{aligned}
\Delta \dot{x} & =V \cos (\psi)-V_{\mathrm{E}} \\
\Delta \dot{y} & =V \sin (\psi)
\end{aligned}
$$

Die Sensorgeschwindigkeiten $\dot{x}_{\mathrm{s}}, \dot{y}_{\mathrm{s}}$ erhält man wieder durch Drehung um den Sensoreinbauwinkel $\theta_{i}$.

Aufgrund der starken nichtlinearen Beziehungen in Bewegungs- und Messmodel wird zur Filterung das Unscented Kalman Filter eingesetzt (Julier and Uhlmann, 2004). Da sich die Messbereiche der Sensoren nicht überschneiden und es auch noch kleine Bereiche gibt, in denen Tracks nicht detektiert werden können muss bei der Auswertung der Detektionswahrscheinlichkeit auch die Unsicherheit von $\boldsymbol{x}_{k}$ berücksichtigt werden. Daher werden gemäß der Verteilung $p\left(\boldsymbol{x}_{k} \mid \mathbf{Z}_{k-1}\right)$ mehrere Stichproben gezogen und geprüft, ob diese innerhalb des Sichtfelds liegen. $P_{\mathrm{D}}$ ergibt sich dann als Mittelwert der Stichproben.

\section{Längenschätzung durch Trackfusion}

Abbildung 4 zeigt die entstandenen Trackverläufe während eines Überholmanövers. Es zeigt sich, dass während des Überholmanövers ein zweiter Track für dasselbe Objekt angelegt wird. Dies liegt daran, dass zu dieser Zeit Messungen von Front und Heck des Fahrzeugs vorliegen, die jedoch auf
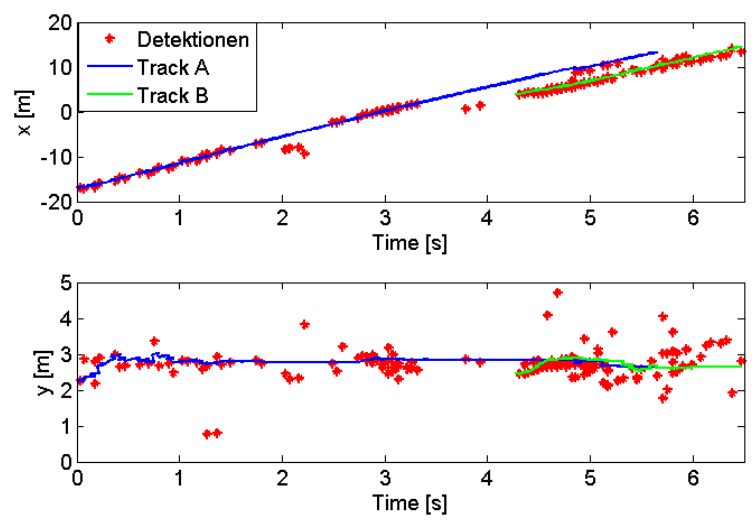

Abb. 4. Positionsschätzung für Fremdfahrzeug bei Überholmanöver in körperfesten Koordinaten des Versuchsfahrzeugs.

Grund des großen Abstands vom GPDA nicht als zusammengehörig erkannt werden. Eine Lösung wäre eine weitere Erhöhung der Messkovarianz, beispielsweise unter der Annahme, dass das Messrauschen ausschließlich durch die Größe des Zielobjekts bestimmt wird. Ein entsprechender Ansatz wird beispielsweise in Koch (2008) präsentiert, jedoch wird hier die Annahme getroffen, dass die Detektionen über die ganze Ausdehnung gleich verteilt sind. Da dies hier nicht der Fall ist, wird ein alternativer Ansatz vorgestellt:

Es wird die Entstehung eines weiteren Tracks grundsätzlich zugelassen, und für den Fall, dass zwei oder mehrere Tracks als ein Objekt klassifiziert werden, diese mittels einer Track-zu-Track Fusion zusammengefasst (Tian and BarShalom, 2009).

Zur Berechnung, ob zwei Tracks zu einem Objekt gehören, wird angenommen, dass ein Objekt eine linienförmige Ausdehnung hat. Für jeden Track wird eine Gerade aus Position und Kurswinkel berechnet. Ergibt sich für mehrere Tracks dieselbe Gerade, haben diese Tracks die gleiche Geschwindigkeit und einen ausreichend kleinen räumlichen Abstand, so werden diese als ein Objekt aufgefasst.

Zur Darstellung eines fusionierten Tracks wird ein neuer Zustandsvektor $\tilde{\boldsymbol{x}}_{k}=[x, y, \psi, r, V, l]$ definiert, welcher das CTRV-Modell um die Länge $l$ eines Fahrzeugs erweitert. Werden erstmalig zwei verschiedene Tracks $\boldsymbol{x}_{k}^{(1)}, \boldsymbol{x}_{k}^{(2)}$ zur Fusion detektiert, wird der neue Zustandsvektor mit der Beziehung

$\tilde{\boldsymbol{x}}_{k}=\left\{\begin{array}{c}\frac{1}{2}\left(\boldsymbol{x}_{k}^{(1)}+\boldsymbol{x}_{k}^{(2)}\right) \\ \left|\left(x^{(1)}-x^{(2)}\right) \cos \tilde{\psi}-\left(y^{(1)}-y^{(2)}\right) \sin \tilde{\psi}\right|\end{array}\right.$

ermittelt. Durch Anwendung der Unscented Transform erhält man für die fusionierte Lösung $\tilde{\boldsymbol{x}}_{k}$ die zugehörige Kovarianzmatrix $\tilde{\mathbf{P}}_{k}$.

Für das nächste Messdaten-Update werden nun die Einzeltracks und die fusionierte Lösung prädiziert. Nach dem Messdatenupdate der Einzeltracks wird erneut geprüft, ob die Zuordnung zum fusionierten Track gültig ist. Wenn dies 

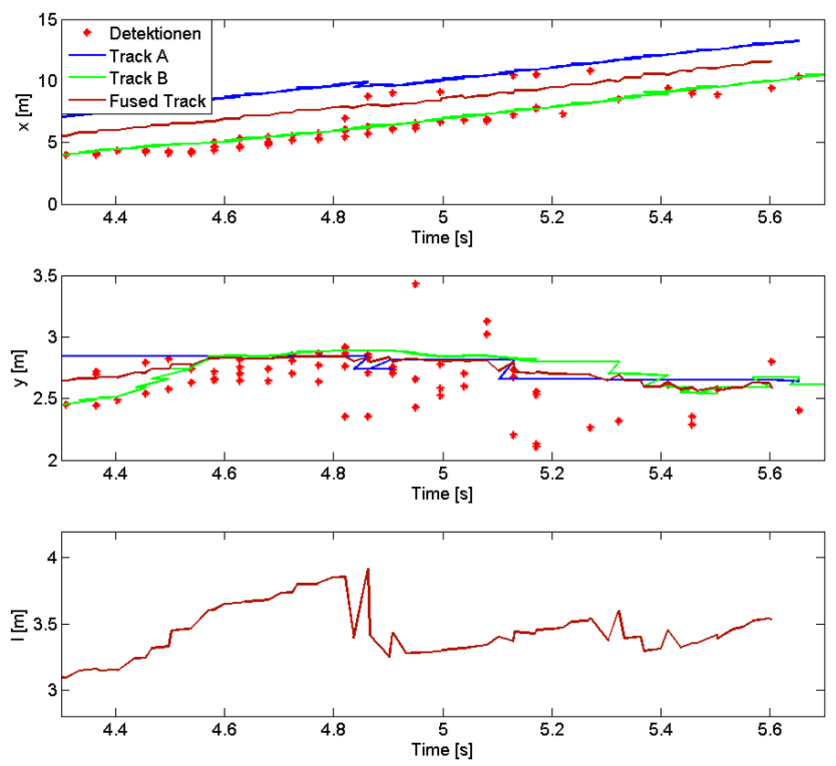

Abb. 5. Fusionierte Positions- und Längenschätzung

der Fall ist, wird die fusionierte Lösung aktualisiert. Dies geschieht unter der Berücksichtigung, dass die einzelnen Tracks bezüglich des Prozessrauschens im Bewegungsmodell korreliert sind. Für die prädizierten Einzeltracks und für die aktualisierten Einzeltracks wird die Fusionlösung $\hat{\mathbf{x}}_{k \mid k-1}, \hat{\mathbf{P}}_{k \mid k-1}$ bzw. $\hat{\mathbf{x}}_{k}, \hat{\mathbf{P}}_{k}$ wieder mittels Unscented Transfrom bestimmt. Damit folgt für den fusionierten Track die neue Kovarianz mit

$\tilde{\mathbf{P}}_{k}^{-1}=\hat{\mathbf{P}}_{k}^{-1}-\hat{\mathbf{P}}_{k \mid k-1}^{-1}+\tilde{\mathbf{P}}_{k \mid k-1}^{-1}$

und der Erwartungswert ist gegeben durch

$\tilde{\boldsymbol{x}}_{k}=\tilde{\mathbf{P}}_{k}\left(\hat{\mathbf{P}}_{k}^{-1} \hat{\mathbf{x}}_{k}-\hat{\mathbf{P}}_{k \mid k-1}^{-1} \hat{\mathbf{x}}_{k \mid k-1}+\tilde{\mathbf{P}}_{k \mid k-1}^{-1} \tilde{\boldsymbol{x}}_{k \mid k-1}\right)$.

Der gezeigte Ansatz kann leicht auf beliebig viele Einzeltracks erweitert werden. Die Einzeltracks bleiben unabhängig, wodurch eine mögliche Falschfusion keine Auswirkungen auf spätere Zeitpunkte hat. Wenn die Existenzwahrscheinlichkeit eines Einzeltracks zu gering ist oder die $\mathrm{Zu}$ ordnungsbedingung nicht mehr erfüllt ist, wird die fusionierte Tracklösung verworfen.

Abbildung 5 zeigt den Verlauf der fusionierten Tracklösung sowie die zugeordnete Längenschätzung eines Fahrzeugs mit einer Gesamtlänge von ca. $3.9 \mathrm{~m}$. Im Mittel bleibt damit die Schätzung in diesem Beispiel etwa $15 \%$ hinter dem wahren Wert zurück. Dies begründet sich in erster Linie durch die Lage der Radarreflektionspunkte. Während des Überholvorgangs werden die dominanten Reflektionen primär von den Radhäusern des Zielfahrzeugs erzeugt. Aufgrund der Sensoranordnung ist es zudem zu keinem Zeitpunkt möglich Messungen gleichzeitig von Front- und Heckstoßfänger zu erhalten. Dies führt dazu, dass das Fahrzeug zumeist etwas kürzer wahrgenommen wird.

\section{Fazit}

Es wurde ein Konzept zur Rundumüberwachung eines Fahrzeugs mittels Radar vorgestellt. Der Ansatz ist primär für die Anwendung bei Spurwechselassistenten ausgelegt, für welche eine stabile Fahrzeugerkennung und Prädiktion möglich ist. Es wurde gezeigt, dass der Effekt der mehreren entstehenden Tracks ausgenutzt werden kann, um mittels einer Track-zu-Track Fusion das Gesamttrackergebnis zu verbessern und eine Schätzung über die Länge des überholenden Fahrzeugs durchzuführen. Die gefundene Länge kann beispielsweise zur Verbesserung der Freiraumschätzung verwendet werden. Da die Track-zu-Track Fusion jedoch nicht optimal im Sinne der Sensorfusion ist, wird zur Zeit ein Ansatz untersucht mit welchem es möglich ist die Radar-Reflektionszentren auf einem Fahrzeug direkt zu verfolgen, und dadurch eine Fusion auf Messdatenebene zu ermöglichen.

Edited by: M. Chandra

Reviewed by: two anonymous referees

\section{Literatur}

Blackman, S. and Popoli, R.: Design and Analysis of Modern Tracking Systems, Artech House Boston, 1999.

Buhren, M. and Yang, B.: Simulation of Automotive Radar Target Lists using a Novel Approach of Object Representation, in: Intelligent Vehicles Symposium, 2006 IEEE, 314-319, 2006.

Darms, M., Rybski, P., Baker, C., and Urmson, C.: Obstacle Detection and Tracking for the Urban Challenge, IEEE T. Intell. Transp., 10, 475-485, 2009.

Erdinc, O., Willett, P., and Bar-Shalom, Y.: Probability hypothesis density filter for multitarget multisensor tracking, in: Information Fusion, 2005 8th International Conference on, 1, 8 pp., 2005.

Julier, S. and Uhlmann, J.: Unscented Filtering and Nonlinear Estimation, P. IEEE, 92, 401-422, 2004.

Koch, J.: Bayesian approach to extended object and cluster tracking using random matrices, IEEE T. Aero Elec. Sys., 44, 1042-1059, 2008.

Musicki, D., Evans, R., and Stankovic, S.: Integrated probabilistic data association, IEEE T. Automat. Contr., 39, 1237-1241, 1994

Schubert, R., Richter, E., and Wanielik, G.: Comparison and evaluation of advanced motion models for vehicle tracking, in: Information Fusion, 2008 11th International Conference on, 1-6, 2008.

Schubert, R., Schulze, K., and Wanielik, G.: Situation Assessment for Automatic Lane-Change Maneuvers, IEEE T. Intell. Transp., 11, 607-616, 2010.

Schubert, R., Adam, C., Richter, E., Bauer, S., Lietz, H., and Wanielik, G.: Generalized probabilistic data association for vehicle tracking under clutter, in: Intelligent Vehicles Symposium (IV), 2012 IEEE, 962-968, 2012.

Tian, X. and Bar-Shalom, Y.: Exact algorithms for four track-totrack fusion configurations: All you wanted to know but were afraid to ask, in: Information Fusion, 2009. FUSION '09. 12th International Conference on, 537-544, 2009. 